\title{
The use of rituximab in idiopathic inflammatory myopathies: description of a monocentric cohort and review of the literature
}

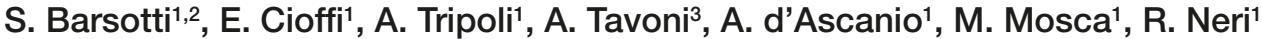 \\ ${ }^{1}$ Rheumatology Unit, University of Pisa, Italy; ${ }^{2}$ Department of Medical Biotechnologies, \\ University of Siena, Italy; ${ }^{3}$ Clinical Immunoallergology, University of Pisa, Italy
}

Conference presentation: LIII SIR Congress (2016).

\section{SUMMARY}

Rituximab (RTX), a chimeric monoclonal antibody targeted against CD20, has been used to treat refractory inflammatory myopathies (IIM).

The primary objective of this study was to retrospectively assess the efficacy of RTX in reducing disease activity in patients with IIM refractory to conventional therapy. Secondary aim was the evaluation of adverse events (AE) during the treatment period.

We examined 26 patients with a diagnosis of IIM, referred to our Rheumatology Unit and treated with RTX for active refractory disease. Patients were treated with RTX $1000 \mathrm{mg}$ i.v., twice, with a 2-week interval.

RTX treatment was associated with a significant reduction of creatine kinase $(\mathrm{p}=0.001)$ after six months compared to the baseline, an improved muscular strength measured with MMT8 $(\mathrm{p}<0.001)$ and a reduction of the extramuscular activity of the disease measured with MYOACT $(\mathrm{p}<0.001)$. In particular, RTX improved DM skin rash, arthritis and pulmonary manifestations. Autoantibody positivity (in particular antisynthetase, antiSRP and antiRo/SSA), and a disease duration $<36$ months at the moment of the treatment are associated with a better response rate. Treatment with RTX was also associated with a reduction of the mean daily dose of steroids needed to control disease activity $(\mathrm{p}=0.002)$.

Our results have confirmed that RTX is efficacious in the treatment of refractory IIM. Ad hoc controlled trials are needed to better clarify the specific subset of patients who may better respond to the treatment and the optimal therapeutic schedule.

Key words: Myositis; Refractory; Rituximab; Polymyositis; Dermatomyositis.

\section{INTRODUCTION}

diopathic inflammatory myopathies (IIMs) are a heterogeneous group of autoimmune diseases characterized by inflammation of the skeletal muscles and involvement of internal organs such as lungs, heart, esophagus and, rarely, kidneys. IIMs are classifiable in different subtypes, the most common are polymyositis (PM), inclusion body myositis (IBM), necrotizing autoimmune myopathy (NAM) and dermatomyositis (DM) (1). Some patients may have a peculiar clinical pattern characterized by arthritis, myositis, and interstitial lung disease (ILD) with positivity for antisynthetase au- toantibodies (ASA) and are further classifiable as antisynthetase syndrome (ASS) (2). Although corticosteroids are usually used as a first line therapy in the treatment of IIM (3), there are no standard therapeutic guidelines for its treatment, particularly due to the rarity of the disease and the presence of different subtypes, making it difficult to design randomized controlled clinical trials (4).

A large number of patients are therefore treated with high cumulative doses of steroids and/or different immunosuppressive drugs to control the disease (3).

Starting from 2005 several case reports (57 ), case series, open label trials (8-10) and 
reports of registries (11) have suggested the efficacy of treatment with RTX in patients with IIM. Recently, two randomized controlled trials (RCTs) have been published $(12,13)$ with controversial results. In the first RCT, the Rituximab in Myositis (RIM) trial, while the trial failed to achieve the primary efficacy endpoint $(12,14,15)$, RTX was able to reduce the clinical activity and the daily prednisone dose taken by refractory patients. In the second trial, conducted in 12 patients with ASA positivity, RTX was reported to be efficacious on lung and muscular involvement (13).

The primary aim of our study was to evaluate the efficacy of RTX in a monocentric cohort of patients with refractory IIM. The secondary aim was to evaluate the adverse events (AE) occurring during the treatment.

\section{MATERIALS AND METHODS}

We retrospectively evaluated consecutive in- and out-patients with a definite diagnosis of PM or DM according to the Bohan and Peter criteria $(16,17)$ or a diagnosis of IBM according to the Griggs criteria (18). Patients treated with RTX for refractory disease, defined as a disease that did not improve with corticosteroids and at least one immunosuppressant, were included in the study.

Treatment schedule was represented by RTX $1000 \mathrm{mg}$ Iv twice, with a 2-week interval. Retreatment with RTX was prescribed in case of disease relapse and after at least 6 months from the previous infusion.

At baseline and 6 months after treatment, activity measures included in the International Myositis Assessment \& Clinical Studies Group (IMACS) core set were evaluated (15):

1) evaluation of global muscle strength with manual muscle testing 8 (MMT8) (16);

2) disease activity using the myositis disease activity assessment visual analogue scales (MYOACT) (17) to obtain the single score of the different subscales (constitutional, cutaneous, skeletal, gastrointestinal, pulmonary and cardiovascular) and the global score;
3) physicians' global activity assessment by a $10 \mathrm{~cm}$ VAS (pVAS) (18);

4) creatine kinase (CK) levels.

Patients' sera were tested for antinuclear autoantibody (ANA) and for extractable nuclear antigens (ENA) by counter immunoelectrophoresis for sera collected between 2005 and 2014; the only myositis specific antibodies (MSA) available were anti-Jo1, while myositis associated antibodies (MAA) available were anti-Ku and anti-SSA. Starting from 2015, sera were also tested for MSA and MAA by line blot (Myositis profile 3, Euroimmun, Lubeck) and the following specificity were available: Mi-2, Ku, PM-Scl100, PM-Scl75, Jo1, SRP, PL-7, PL-12, EJ, OJ, Ro-52.

The efficacy of the treatment was assessed after 6 months, and was evaluated according to a simplified version of IMACS definition of improvement (19). The improvement was defined as major if the patient showed an increase of at least $15 \%$ in muscle strength by MMT8 and 30\% reduction in CPK after 6 months (20). The improvement was defined as minor if the patient obtained improvement in at least 2 of the four IMACS core set measures: $15 \%$ improvement in total MMT8, 20\% improvement in pVAS, $20 \%$ improvement in the MYOACT score, $30 \%$ reduction of CK (20). No more than 1 measure should worsen by $\geq 25 \%$ and the MMT could not be one of the worsening measures.

To evaluate the toxicity, the appearance of $\mathrm{AE}$ was scored according to common terminology criteria for adverse events (CTCAE) v4.0 according to the following scale: grade 1: mild, grade 2: moderate; grade 3: severe, grade 4: very severe. All the adverse events not attributable to any other disease or concomitant medication were taken into account. AE were classified as directly related to treatment if the EA was associated with the mechanism of action of the drug (e.g. infections), or not directly related to the treatment if the EA was not expected and/or related to allergic sensitization (e.g. sneezing, difficulty in breathing, cramps, and vomiting).

The following data were also collected: indications to start treatment, previous and 
concomitant therapies, disease-free interval, number and timing of retreatment.

\section{Statistical analysis}

The variables were expressed as mean +/standard deviation (SD). The difference between baseline and after six months from the therapy was calculated using the parametric test (ANOVA) when the variables were normally distributed or using nonparametric test Mann-Whitney and KruskalWallis when not normally distributed (SPSS statistics 19, IBM). A multivariate analysis was performed to identify predictive factors of the response to the treatment.

\section{RESULTS}

\section{Baseline characteristics}

From September 2005 to September 2016, 26 patients were treated with RTX (9 with DM, 15 with PM, 2 with IBM). Five pa-

Table I - Demographic and clinical data of the patients (baseline). Data are expressed in mean \pm standard deviation or number of patients (\%).

\begin{tabular}{|l|c|}
\hline Number of patients & 26 \\
\hline PM/DM/MCl & $15 / 9 / 2$ \\
\hline Male/Female & $7 / 19$ \\
\hline Age at the treatment mean \pm SD (years) & $55.6 \pm 16.7$ \\
\hline Disease duration at the time of treatment & $41.12 \pm 39.8$ \\
mean \pm SD (months) & \\
\hline Clinical involvement - patients (percentage) & $26(100 \%)$ \\
Reduction of muscular strength & $16(61 \%)$ \\
Dysphagia & $4(16 \%)$ \\
Dyspnea & $9(35 \%)$ \\
DM Skin rash & $7(27 \%)$ \\
Arthritis & \\
\hline Previous treatment & $17.6 \pm 19.7$ \\
Corticosteroid dose - mean $\pm S D$ (grams) & \\
\hline Immunosuppressant - patient (percentage) & $11(42 \%)$ \\
Methotrexate & $5(19 \%)$ \\
Cyclosporin A & $3(12 \%)$ \\
Cyclophosphamide & $2(8 \%)$ \\
Azathioprine & $5(19 \%)$ \\
Mycophenolate mofetil & 13 Jo-1. $1 \mathrm{Pl}-7.1 \mathrm{PI}-12)$ \\
\hline Autoantibodies - patients (percentage) & $1(4 \%)$ \\
Antisynthetase autoantibodies & $1(4 \%)$ \\
Anti-SRP & $7(27 \%)$ \\
Anti-Tif1 $\gamma$ & $1(4 \%)$ \\
Anti-Ro/SSA & $1(4 \%)$ \\
Anti-La/SSB & \\
Anti-RNP & \\
\hline
\end{tabular}

tients were affected by ASS. Clinical and epidemiologic data are reported in Table I. All patients fulfilled the classification criteria. The reason for RTX treatment was the presence of an active disease and all patients presented a pVAS and/or MYOACT global score $\geq 3$.

All patients presented a reduction of muscular strength (8 patients were bedridden), while extra muscular manifestations were dysphagia in 16 patients, dyspnea in 4, DM skin rash in 9 and peripheral arthritis in 7. All the patients had been treated with high dose 6 methylprednisolone (6MP) with an approximate mean cumulative dose of 5.8 grams (min-max 0.2-19). At the time of treatment, the patients were treated with a mean daily dose of $17.6 \pm 19.7 \mathrm{mg}$ of $6 \mathrm{MP}$ and all patients were treated with one immunosuppressive drug (Table I). Seventeen patients were receiving Iv-Ig.

ANA were positive in $13(50 \%)$ patients; anti-ENA antibodies were observed in 13 patients (50\%). Autoantibodies specificities observed are reported in Table I.

\section{Evaluation after six months}

The clinimetric parameters included in the study at baseline and after 6 months from treatment are reported in Table II. Unfortunately, 5 patients did not have the complete data set recorded as they were treated before 2010. The improvement was therefore calculated in only 21 patients.

According to the definition of improvement defined by the International Myositis Assessment \& Clinical Studies Group (IMACS) (15):

1) CK reduced by $30 \%$ in $20 / 26$ patients;

2) MMT8 improved by $15 \%$ in $11 / 22$ patients, stabilized in 11/22 and did not worsen in any patients;

3) MYOACT reduced by $20 \%$ in $18 / 23$ patients;

4) pVAS improved by $20 \%$ in $17 / 24$ patients.

Among the 21 patients with a complete dataset, 8 patients achieved the threshold for major improvement as defined in the methods section, while 7 patients reached the threshold for minor improvement.

The improvement of extramuscular mani- 
festations, evaluated with the cutaneous, skeletal, gastrointestinal, pulmonary subscale of MYOACT, are reported in Table II. Particularly, DM skin rash improved in $8 / 9$ patients, arthritis in 7/7 patients, dysphagia in $8 / 16$ patients, and dyspnea in $7 / 8$ patients. After the treatment only 3 patients (1 with PM, 2 with IBM) were bedridden. No statistically significant difference between PM and DM was found.

A higher number of patients with major improvement was observed in the 11 patients with autoantibodies positivity (ASS and/or anti-Ro/SSA and/or antiSRP) compared to the other 15 seronegative patients $(\mathrm{p}=0.036)$. In particular, all the 5 patients with ASS reached a major improvement and presented clinical response for muscular, articular and respiratory involvement. The patient with anti-SRP positivity and 5/7 patients with anti-Ro/SSA positivity also showed a muscular response to the treatment. On the contrary, the two patients with IBM, one of them with anti-Ro/SSA positivity, did not show clinical improvement, in particular for muscular involvement and dysphagia.

The response rate seems to be inversely associated with disease duration: the patients with a major improvement had a lower mean disease duration $(18.4 \pm 20.42$ months) compared to patients with minor improvement $(42.5 \pm 31.6$ months $\mathrm{p}=0.045)$ and those that did not improve $(48.8 \pm 43.9$ months $\mathrm{p}=0.04$ ). Moreover, major improvement was observed in $57.1 \%$ of patients with a disease duration lower than 36 months compared to only $11.1 \%$ of patients with a disease duration higher than 36 months $(\mathrm{p}=0.04)$.

With multivariate analysis, the disease duration was the only independent factor for predicting response to RTX in our cohort (OR 0.9, 95\% CI 0.82-0.99, $\mathrm{p}=0.03$ ).

In addition to the clinical response, there was a reduction in the mean daily corticosteroid dose in 20/26 patients, from $17.6 \pm 19.7 \mathrm{mg} / \mathrm{die}$ to $4.9 \pm 2.2 \mathrm{mg} / \mathrm{die}$ $(\mathrm{p}=0.002)$.

\section{Adverse events}

AEs were observed in 5 patients $(19 \%)$ and 3 patients (12\%) discontinued RTX be-
Table II - Clinimetric evaluation at baseline and after 6 months from the treatment.

\begin{tabular}{|l|c|c|c|}
\hline Parameter & Baseline (mean \pm SD) & 6 months $($ mean \pm SD) & $\mathbf{p}$ \\
\hline CK (UI/L) & $2261 \pm 2675$ & $385 \pm 483$ & 0.001 \\
\hline MMT8 & $56.7 \pm 10.4$ & $69.9 \pm 11.0$ & $<0.001$ \\
\hline MYOACT (cm) & & & \\
Global score & $2.4 \pm 1.9$ & $1.3 \pm 0.8$ & $<0.001$ \\
Subscales & $1.97 \pm 2.84$ & $1.21 \pm 2.36$ & 0.014 \\
Cutaneous & $1.25 \pm 2.40$ & $0.32 \pm 0.83$ & 0.025 \\
Skeletal & $2.996 \pm 3.05$ & $2.37 \pm 2.67$ & 0.049 \\
Gastrointestinal & $1.57 \pm 2.50$ & $0.77 \pm 1.34$ & 0.021 \\
Pulmonary & $5.66 \pm 1.76$ & $3.04 \pm 1.58$ & $<0.001$ \\
Constitutional & $0.367 \pm 1.13$ & $0.04 \pm 0.20$ & NS \\
Cardiovascular & $6.5 \pm 1.4$ & $3.3 \pm 1.7$ & $<0.001$ \\
\hline pVAS (cm) & \multicolumn{2}{|c}{} \\
\hline
\end{tabular}

cause of AE, one after the first infusion, 2 after the second infusion. The average time between the first infusion of RTX and the $\mathrm{AE}$ was 17 days (min $4-\max 30$ ) and in four cases the AEs were reported after the second administration of RTX. We recorded 2 AEs directly related to the treatment and 3 not directly related to the treatment. According to CTCAE classification, a grade 2 event was observed in 1 patient, and grade 3 in 4 patients. Descriptions of the AEs, severity and the treatment are reported in Table III.

\section{Last follow up}

In December 2016, twenty-one patients were still in follow-up. The mean followup duration was $48.6 \pm 38.8$ months.

During the follow-up, 8 responder patients relapsed on average after $17 \pm 18$ months from the treatment. All the relapsed patients were successfully retreated with RTX.

Among the 5 patients who did not continue the follow up: 1 patient was lost to follow-up and four patients died for diseaserelated causes with a mean disease duration of 80.7 months ( $\min 23, \max 144)$. The disease-related causes were: respiratory failure due to muscle weakness in a patient with IBM, heart involvement in two DM patients (one for cardiac sudden death and one for heart insufficiency), severe pneumonia in one patient with PM. The average time between the last infusion of RTX and the death was 45 months (min 14 - max 94). 
Table III - Adverse events, classification, grade and treatment.

\begin{tabular}{|c|l|l|l|c|}
\hline Event & \multicolumn{1}{|c|}{ CTCAE system organ class } & \multicolumn{1}{c|}{ Description } & \multicolumn{1}{c|}{ Treatment } & Grade (range 1-5) \\
\hline 1 & Infections and infestations & Bladder infection & Oral antibiotics & 2 \\
\hline 2 & Infections and infestations & CMV infection & Antiviral drugs, oxygen support & 3 \\
\hline 3 & Immune system disorder & Allergic reaction & Steroids and IV liquids & 3 \\
\hline 4 & Immune system disorder & Allergic reaction & Steroids and IV liquids & 3 \\
\hline 5 & $\begin{array}{l}\text { Skin and subcutaneous } \\
\text { tissue disorders }\end{array}$ & Erythema multiforme & IV steroids & 3 \\
\hline
\end{tabular}

\section{DISCUSSION AND CONCLUSIONS}

Literature data show a strong evidence for a critical role of B cells in IIM, in particular for DM $(21,22)$ as well as, more recently, in the pathogenesis of $\mathrm{PM}$ and IBM (23). The results of our study confirm the efficacy of RTX in the treatment of refractory IIM. The treatment was effective both in muscular and in some extramuscular manifestations of the disease. Prognostic factors for a higher response rate were the autoantibody positivity (antisynthetase autoantibody, anti-SRP and anti-Ro/SSA). The extramuscular manifestations that showed an improvement after the treatment were articular, skin and respiratory involvement, while dysphagia improved only in a small group of patients. Patients with IBM did not improve with the treatment. The treatment of patients with shorter disease duration seems to be associated with a better outcome, in particular when treatment was started in patients with a disease duration shorter than 36 months.

Literature data confirm our observations about the good efficacy in DM patients (24-27), in particular in patients with the juvenile form (28). Several studies also reported a good outcome in patients with lung disease, in particular when associated with antisynthetase autoantibodies positivity $(13,29-34)$. No clear data are available in medical literature about the efficacy of RTX in articular and esophageal manifestations on IIM and in the treatment of IBM. Our observations about a better prognosis in patients with autoantibodies positivity are consistent with the literature, in par- ticular when associated with antisynthetase autoantibodies positivity $(5,7,28,31,33$, $35)$. Also anti-SRP positivity was reported to be associated to a better response to RTX treatment $(36,37)$. In a recent publication on 17 patients with anti-SRP associated myositis, $13 / 17$ patients improved after the treatment (38).

We observed, in our population, that a good outcome may be reached in patients with a disease duration lower than three years. This aspect is partially in contrast with other reports in medical literature, where some authors described a better response in patients with a disease duration of less than 1 year (30). In addition, in our study, multivariate analysis identified disease duration as the only independent factor to predict the response to RTX.

In our cohort of patients RTX was also effective in reducing the corticosteroid daily dose, and these data are consistent with other studies $(13,39)$. Patients with IIM are often treated with high cumulative corticosteroids doses and a reduction of the daily dose may lower the incidence of side effects, such as the steroid myopathy (40). We also observed one moderate and four severe AEs: we have reported two infections, two immune-mediated reactions and one skin rash. A higher prevalence of these AEs in IIM patients was also reported by other authors; in particular, infusion reactions $(35,39,41)$ and infections have been correlated to treatment with RTX. Although infections during treatment with RTX in IIM patients were usually mild (39), rare cases of severe AEs have been reported including viral and bacterial infections (41, 42) and few cases of multifocal leukoencephalopathy $(43,44)$. 
The main point of strength of our work is the large monocentric group of patients with IIM treated with RTX and the long duration of the follow-up, although the main limitation is the retrospective data collection.

Although the safety profile does not seem free from adverse events, RTX certainly represents a new perspective for the therapeutic approach of IIM. Further ad hoc controlled trials are needed to better clarify the specific subset of patients associated with a higher response rate to the treatment and the specific schedule of management.

\section{REFERENCES}

1. Dalakas MC. Inflammatory muscle diseases. N Engl J Med. 2015; 372: 1734-47.

2. Cavagna L, Nuño L, Scirè CA, et al. Clinical spectrum time course in anti jo-1 positive antisynthetase syndrome: Results from an international retrospective multicenter study. Med (United States). 2015; 94.

3. Oddis CV. Update on the pharmacological treatment of adult myositis. J Intern Med. 2016; 280: 63-74

4. Moghadam-Kia S, Oddis CV, Aggarwal R. Modern Therapies for Idiopathic Inflammatory Myopathies (IIMs): Role of Biologics. Clin Rev Allergy Immunol. 2017; 52: 81-7.

5. Brulhart L, Waldburger J-M, Gabay C. Rituxi$\mathrm{mab}$ in the treatment of antisynthetase syndrome. Ann Rheum Dis. 2005; 65: 974-5.

6. Chiappetta N, Steier J, Gruber B. Rituximab in the treatment of refractory dermatomyositis. J Clin Rheumatol. 2005; 11: 264-6.

7. Lambotte O, Kotb R, Maigne G, et al. Efficacy of rituximab in refractory polymyositis. J Rheumatol. 2005; 32: 1369-70.

8. Levine TD. Rituximab in the treatment of dermatomyositis: An open-label pilot study. Arthritis Rheum. 2005; 52: 601-7.

9. Mok CC, Ho LY, To CH. Rituximab for refractory polymyositis: an open-label prospective study. J Rheumatol. 2007; 34: 1864-8.

10. Chung L, Genovese MC, Fiorentino DF. A Pilot Trial of Rituximab in the Treatment of Patients With Dermatomyositis. Arch Dermatol. 2007; 143: 763-7.

11. Couderc M, Gottenberg J-E, Mariette X, et al. Efficacy and safety of rituximab in the treatment of refractory inflammatory myopathies in adults: results from the AIR registry. Rheumatology 2011; 50: 2283-9.

12. Oddis CV, Reed AM, Aggarwal R, et al. Rituximab in the treatment of refractory adult and juvenile dermatomyositis and adult polymy- ositis: a randomized, placebo-phase trial. Arthritis Rheum. 2013; 65: 314-24.

13. Allenbach Y, Guiguet M, Rigolet A, et al. Efficacy of Rituximab in Refractory Inflammatory Myopathies Associated with Anti-Synthetase Auto-Antibodies: An Open-Label, Phase II Trial. PLoS One. 2015; 10: e0133702.

14. de Visser M. Editorial: The efficacy of rituximab in refractory myositis: The jury is still out. Arthritis Rheum. 2013; 65: 303-6.

15. Rider LG, Giannini EH, Harris-Love M, et al. Defining Clinical Improvement in Adult and Juvenile Myositis. J Rheumatol. 2003; 30: 603-17.

16. Rider LG, Koziol D, Giannini EH, et al. Validation of manual muscle testing and a subset of eight muscles for adult and juvenile idiopathic inflammatory myopathies. Arthritis Care Res (Hoboken). 2010; 62: 465-72.

17. Isenberg DA, Allen E, Farewell V, et al. International consensus outcome measures for patients with idiopathic inflammatory myopathies. Development and initial validation of myositis activity and damage indices in patients with adult onset disease. Rheumatology. 2004; 43: 49-54.

18. Rider LG, Feldman BM, Perez MD, et al. Development of validated disease activity and damage indices for the juvenile idiopathic inflammatory myopathies: I. Physician, parent, and patient global assessments. Juvenile Dermatomyositis Disease Activity Collaborative Study Group. Arthritis Rheum. 1997; 40: 1976-83.

19. Rider LG, Giannini EH, Brunner HI, et al. International consensus on preliminary definitions of improvement in adult and juvenile myositis. Arthritis Rheum. 2004; 50: 2281-90.

20. Rider LG, Giannini EH, Harris-Love M, et al. Defining Clinical Improvement in Adult and Juvenile Myositis. J Rheumatol. 2003; 30: 603-17.

21. Lemoine NR, Ryan JF, Cox EL, et al. Immunohistochemical analysis of mononuclear cell subsets in inflammatory and non-inflammatory myopathies. J Clin Pathol. 1986; 39: 271-4.

22. Grundtman C, Malmström V, Lundberg IE. Immune mechanisms in the pathogenesis of idiopathic inflammatory myopathies. Arthritis Res Ther. 2007; 9: 208.

23. Greenberg SA, Bradshaw EM, Pinkus JL, et al. Plasma cells in muscle in inclusion body myositis and polymyositis. Neurology. 2005; 65: 1782-7.

24 Aggarwal R, Loganathan P, Koontz D, et al. Cutaneous improvement in refractory adult and juvenile dermatomyositis after treatment with rituximab. Rheumatology. 2017; 56: 247-54.

25. Haroon M, Devlin J. Rituximab as a first-line agent for the treatment of dermatomyositis. Rheumatol Int. 2012; 32: 1783-4. 
26. Parziale N, Kovacs SC, Thomas CB, et al. Rituximab and Mycophenolate Combination Therapy in Refractory Dermatomyositis With Multiple Autoimmune Disorders. J Clin Neuromuscul Dis. 2011; 13: 63-7.

27. Joshi N, Nautiyal A, Davies PG. Successful Use of Rituximab in Recalcitrant Skin Predominant Dermatomyositis. J Clin Rheumatol. 2011 ; 17 : 111-2.

28. Aggarwal R, Bandos A, Reed AM, et al. Predictors of Clinical Improvement in Rituximab-Treated Refractory Adult and Juvenile Dermatomyositis and Adult Polymyositis. Arthritis Rheumatol. 2014; 66: 740-9.

29. Sharp C, McCabe M, Dodds N, et al. Rituximab in autoimmune connective tissue diseaseassociated interstitial lung disease. Rheumatology. 2016; 55: 1318-24.

30. Andersson H, Sem M, Lund MB, et al. Longterm experience with rituximab in anti-synthetase syndrome-related interstitial lung disease. Rheumatology. 2015; 54: 1420-8.

31. Marie I, Dominique S, Janvresse A, et al. Rituximab therapy for refractory interstitial lung disease related to antisynthetase syndrome. Respir Med. 2012; 106: 581-7.

32. Dasa O, Ruzieh M, Oraibi O. Successful Treatment of Life-Threatening Interstitial Lung Disease Secondary to Antisynthetase Syndrome Using Rituximab. Am J Ther. 2016; 23: e639-45.

33. Vandenbroucke E, Grutters JC, Altenburg J, et al. Rituximab in life threatening antisynthetase syndrome. Rheumatol Int. 2009; 29: 1499-502.

34. Furlan A, Botsios C, Ruffatti A, et al. Antisynthetase syndrome with refractory polyarthritis and fever successfully treated with the IL-1 receptor antagonist, anakinra: A case report. Joint Bone Spine Rev Rheum. 2008; 75: 366-7.

35. Nalotto L, Iaccarino L, Zen M, et al. Rituxi$\mathrm{mab}$ in refractory idiopathic inflammatory myopathies and antisynthetase syndrome: per- sonal experience and review of the literature. Immunol Res. 2013; 56: 362-70.

36. Luca NJC, Atkinson A, Hawkins C, et al. Anti-signal Recognition Particle-positive Juvenile Polymyositis Successfully Treated with Rituximab. J Rheumatol. 2012; 39: 1483-5.

37. Valiyil R, Casciola-Rosen L, Hong G, et al. Rituximab therapy for myopathy associated with anti-signal recognition particle antibodies: A case series. Arthritis Care Res (Hoboken). 2010; 62: 1328-34.

38. Pinal-Fernandez I, Parks C, Werner JL, et al. Longitudinal Course of Disease in a Large Cohort of Myositis Patients With Autoantibodies Recognizing the Signal Recognition Particle. Arthritis Care Res (Hoboken). 2017; 69: 263-70.

39. Basnayake C, Cash K, Blumbergs P, et al. Use of rituximab in histologically confirmed idiopathic inflammatory myositis: a case series. Clin Rheumatol. 2015; 34: 371-7.

40. Pereira RMR, de Carvalho J. Glucocorticoidinduced myopathy. Joint Bone Spine Rev Rheum. 2011; 78: 41-4.

41. Unger L, Kampf S, Luthke K, et al. Rituximab therapy in patients with refractory dermatomyositis or polymyositis: differential effects in a real-life population. Rheumatology. 2014; 53: $1630-8$.

42. Nalotto L, Iaccarino L, Zen M, et al. Rituximab in refractory idiopathic inflammatory myopathies and antisynthetase syndrome: personal experience and review of the literature. Immunol Res. 2013; 56: 362-70.

43 Belhassen-Garcia M, Rabano-Gutierrez A, Velasco-Tirado V, et al. Atypical Progressive Multifocal Leukoencephalopathy in a Patient with Antisynthetase Syndrome. Intern Med. 2015; 54: 519-24.

44. Marie I, Guegan-Massardier E, Levesque H. Progressive multifocal leukoencephalopathy in refractory polymyositis treated with rituximab. Eur J Intern Med. 2011; 22: e13-4. 